\title{
Tip-replaceable Horn with Full Wavelength for Ultrasonic Welding
}

\author{
Mi Ae Kim , Chang Seo Im and Dong Sam Park \\ Dept. of Mechanical Engineering, Incheon National Univ., Yeonsu Gu, Incheon, 22012, Korea
}

\begin{abstract}
Ultrasonic welding is faster, environmental friendly, and more efficient than conventional welding. Ultrasonic welding stack, the key part in ultrasonic welding equipment, is composed of transducer, booster, and horn. In the case of ultrasonic welding, wear in the welding tips or breakage of horn occurs because ultrasonic vibration of tips is transmitted directly to the welding material. If grinding of the tips is repeated several times, the mass of horn is reduced and the frequency characteristic is changed. So, there is a limit to refurbishment. In additions, as materials of horn are relatively expensive, some cost problems could be occurred for replacing horns. This study, in order to reduce the manufacturing cost of horn, devised a tip-replaceable ultrasonic horn so that its tip can be easily replaced whenever it is worn. For the designed shape, natural frequency and vibration mode are analyzed using FEA. Through the FEA, the optimal tip-replaceable horn with resonance frequency of $40 \mathrm{kHz}$ is proposed.
\end{abstract}

\section{Introduction}

Ultrasonic welding is a faster, more environmentally friendly and simpler, more efficient welding method than conventional welding. In particular, ultrasonic metal welding has the advantage of being able to bond dissimilar metals such as aluminum and copper [1]. In addition, it is an essential technology for miniaturization and weight reduction of electrical products and automobile manufacturing [2] and is known as the most suitable method for electrode terminal welding of rechargeable batteries used in automobiles and mobile phones.

When two metals are brought into contact and one metal is vibrated in parallel, they are bonded in a short time [3]. Only the vibrational energy is used and no current or external heating is required [4]. Welding occurs at relatively low temperatures $\left(<300 \circ C^{\circ}\right)$ [5], and high-frequency shear vibrations applied under appropriate pressure also have the advantage of removing oxide and contaminant layers [6].

Ultrasonic welding stack, a key component of ultrasonic welding equipment, consists of transducer, booster and horn. The tips knurled in the horn main body require direct contact with the welding material and transmit ultrasound vibrations, which causes tip wear during welding. In this case, re-grinding decreases the mass of the horn and changes the frequency characteristics. Therefore, there is a limit to re-grinding. Also, horns are made of expensive materials such as titanium alloys, which can be expensive if we are to replace horns.

To reduce the cost of the horn, a patent for an ultrasonic horn that can be mounted on the body of the horn in the form of a tip plate has been submitted in Korea [7]. Nissan Motors in Japan also applied for a patent regarding an ultrasonic welding horn that allows the tip to be attached and detached by screws between them. Branson, USA, applied for a patent for an ultrasonic welding horn that can replace a tip using a collet. However, most of these patents relate to half-wave length horns. Although a one-wave replaceable horn has been patented in Japan [8], but its structure is designed to replace almost half of the horn. To overcome this problem, a new horn design is required to minimize the mass of the replacement part and increase efficiency. However, despite the importance of tip-replaceable horns, there is not much academic study of them till now. In this study, we designed a tip-replaceable ultrasonic welding horn with resonance frequency of 40 $\mathrm{kHz}$ and analyzed vibration characteristics using FEA (finite element analysis).

\section{Designing a tip-replaceable horn}

\subsection{Initial model of tip-replaceable horn}

Fig. 1(a) is a 3D model of conventional one-body horn for ultrasonic welding, and Fig. 1(b) is an initial design of the tip-replaceable horn proposed in this study. One of the tip parts is dug out from the conventional metal welding horn, and a rectangular shaped tip is bolted to the horn body. The width of the space for the assembled tip is $18.9 \mathrm{~mm}$, equal to the width of the assembled tip, and its depth is $3.9 \mathrm{~mm}$, equal to the thickness of the tip. So, the left and right sides of the replaceable part are in contact with the horn, and the upper surface of the assembled tip and surface of the horn are both in the same plane. 


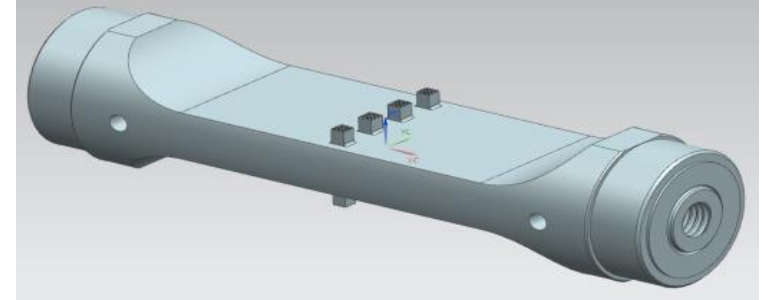

(a) 3D model of a conventional horn

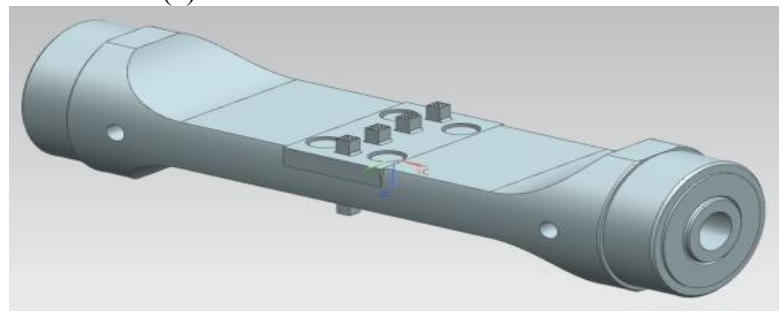

(b) Initial model of the tip-replaceable horn

Figure 1. Models of conventional horn and the tip-replaceable horn proposed in this study

\subsection{Analysis of vibration characteristics}

Finite element analysis was carried out using the tip-replaceable horn model shown in Fig. 1(b). Modal analysis was conducted to analyze natural frequency and vibration mode using the ANSYS tool. Horn material was assumed to be ASP60, and its material properties are ; density $7.9 \mathrm{~g} / \mathrm{cm}^{3}$, Poisson's ratio 0.3 , and Young's modulus $222 \mathrm{Gpa}$.

As contact conditions, "Bonded" was applied to parts that fixed with threaded bolts, and "No Separation" was applied to the parts that were not completely fixed. As support conditions, "Cylindrical support" was applied to bolt hole and the degree of freedom was given only in the axial direction. Vibration modes from 1st to 20th were analyzed. The 14th mode showing the first longitudinal vibration mode is shown in Fig. 2. Its natural frequency is $38,067 \mathrm{~Hz}$, which is about $0.5 \mathrm{kHz}$ less than the natural frequency $(38,526 \mathrm{~Hz})$ of the one-body horn.

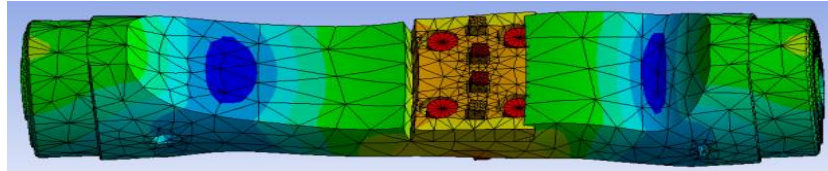

Figure 2. 14th vibration mode (natural frequency 38,067 $\mathrm{Hz})$

\section{Improving horn design by modal analysis}

\subsection{Vibration mode of the initial model}

As there are no contact conditions between the right and left sides of the tip and the horn body, the analysis was carried out without any condition in those parts. In consequence, Interference occurred between tip and horn body as shown in Fig. 2. This indicates that the vibrations of the tip and horn are not exactly the same, and interference may occur between the tip and the horn during vibration. In this case, a continuous impact is applied to both the tip and the horn, which can affect the life of the horn and the quality of the weld.

As shown in Fig. 3(a), the initial model is modified with gap $0.5 \mathrm{~mm}$ between the tip and the horn. Although the vibration mode 14th of the modified model is similar to the longitudinal vibration mode, as shown in Fig. 3(b), vertical bending vibration occurs slightly. In other words, when the transducer or the booster is assembled on the end surfaces of the horn, its vibration may not be properly transmitted because both surfaces do not contact perfectly. It is considered that slight bending vibration is caused by the problem that the upper and lower shapes of the horn are not completely symmetrical. Fig. 3(c) shows the distribution of the vibration amplitude at the cross section along the tips. The amplitude in the replaceable tips is found to be smaller.

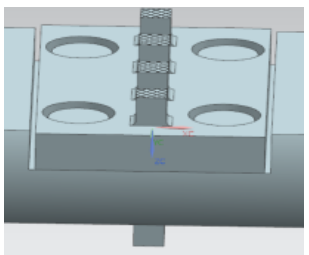

(a) The model with $0.5 \mathrm{~mm}$ gap

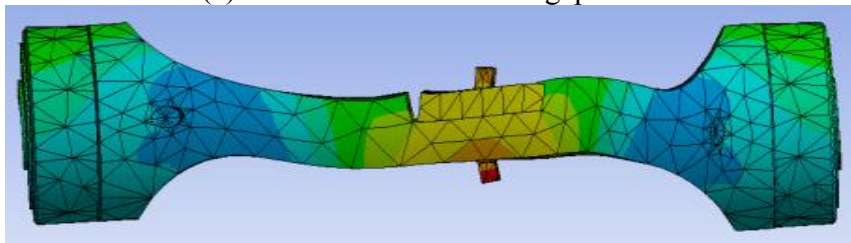

(b) 14th vibration mode

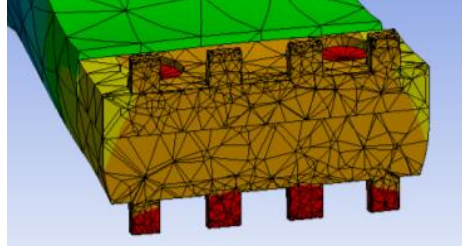

(c) Amplitude distributions in cross section along tips

Figure 3. Modal analysis of the modified horn with $0.5 \mathrm{~mm}$ gaps

\subsection{Improving horn design}

As reduction of the amplitude in the assembled tips and uneven amplitude at the side surfaces of the horn are due to the asymmetry shape of the upper and lower sides of the horn, the horn is modified as shown in Fig. 4. Fig. 4(a) shows the horn that top and bottom tips are replaceable. Fig. 4(b) shows the horn that only the upper tip is replaceable, and in the lower part slots are machined to keep the horn as symmetric as possible.

Fig. 5(a) and Fig. 5(b) show vibration modes of Fig. 4(a) and Fig. 4(b), respectably. Both horns show good longitudinal vibration mode, and the amplitude of replaceable tips is relatively large. The vibration amplitude of end-sides of the horn is relatively uniform. In this study, the horn with one replaceable tips is adopted as a final model considering the rigidity of the horn and the convenience of machining. 
As shown in Fig. 5(b), however, the natural frequency of the $14^{\text {th }}$ mode was $37,281 \mathrm{~Hz}$, which is much lower than that of the conventional one-body type horn, $38,526 \mathrm{~Hz}$. In order to increase the natural frequency, each thickness of the lower and upper sides of the horn was increased by 1 $\mathrm{mm}$. The total depth of slot in lower side is $3.5 \mathrm{~mm}$. Fig. 6(a) shows the horn model modified again, in which the thickness of the area marked in orange is increased. Fig. $6(\mathrm{~b})$ shows the $14^{\text {th }}$ mode shape with a natural frequency of $38,331 \mathrm{~Hz}$. It is confirmed that the mode shape of the modified horn is nearly symmetrical due to the reduction of the protrusion of the tip, so that the vibration occurs almost in the axial direction. The natural frequency of the 14th mode is increased similarly to that of the conventional one-body horn by increasing the mass of the horn.

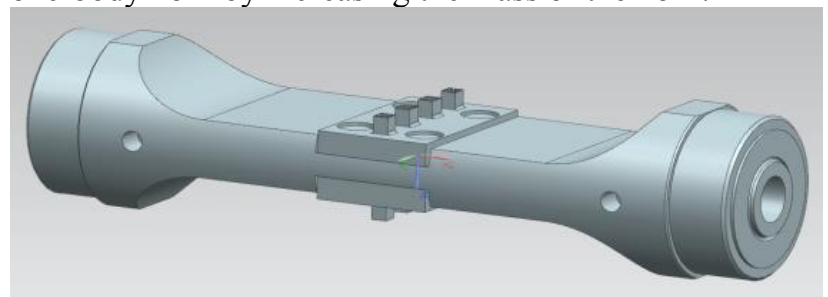

(a) Horn with two replaceable tips

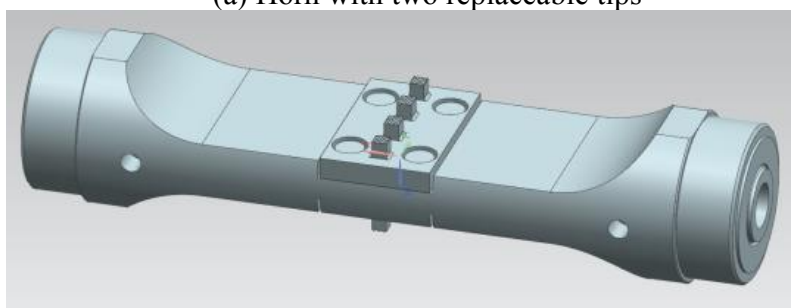

(b) Horn with one replaceable tips

Figure 4. New designs for improving the symmetry

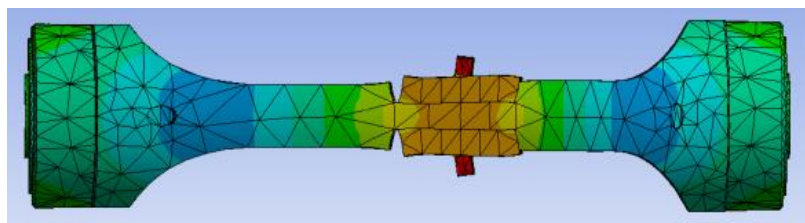

(a) Model of Fig. 4(a) (14th mode, 36,789 Hz)

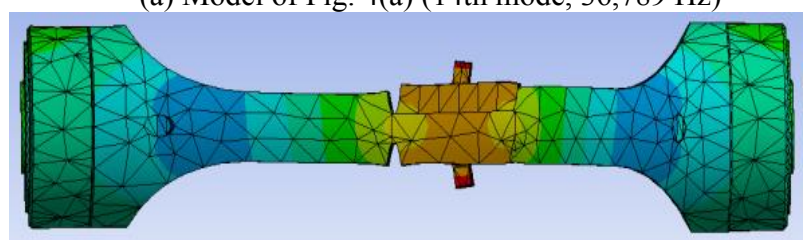

(b) Model of Fig. 4(b) (14th mode, 37,281 Hz)

Figure 5. Vibration modes of new designs

\section{Final design and vibration analysis}

\subsection{Final design}

In the process of finding the optimal design of one tip-replaceable horn, it is confirmed that change of the gap width has almost no effects on the vibrational mode shapes. So the gap between tip and horn is designed to be $2 \mathrm{~mm}$ wide to prevent interference between them. Machined corners are rounded to avoid stress concentration. In the final design, two holes are formed to facilitate pushpin insertion. Two pushpins are used for tight fastening of replaceable tips and horn body in a specified position. Pins are assumed to be assembled without any counterbores on the drilled holes, because there are no significant differences in the natural frequency and mode shape.

The $14^{\text {th }}$ vibration mode and natural frequency of the final model proposed in this study are shown in Fig. 7(a). It is confirmed that vibrations occur almost in the axial direction and the natural frequency is similar to that of the conventional one-body horn. Fig. 7(b) shows distributions of the vibration amplitude at the cross section along joining bolts. It is confirmed that vibrational amplitudes are maximum at the tips and are nearly symmetrical in the upper and lower sides. These results imply that the final design for the tip-replaceable horn could be applied to ultrasonic welding effectively.

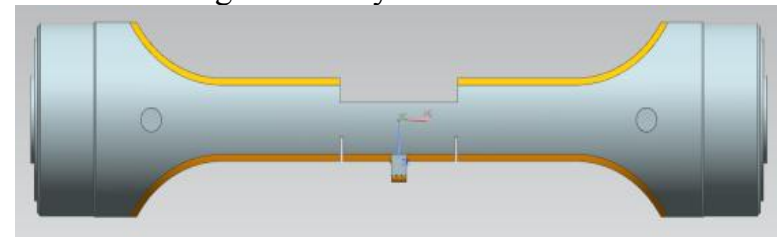

(a) Modified area

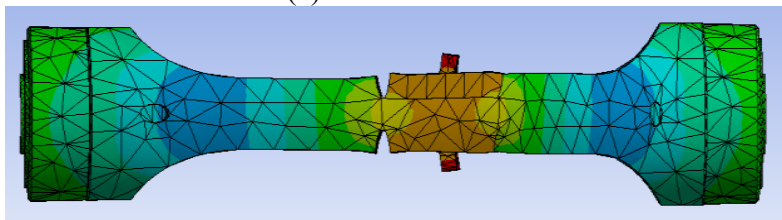

(b) 14th mode shape of the redesigned model

Figure 6. Redesigned model and the $14^{\text {th }}$ mode shape

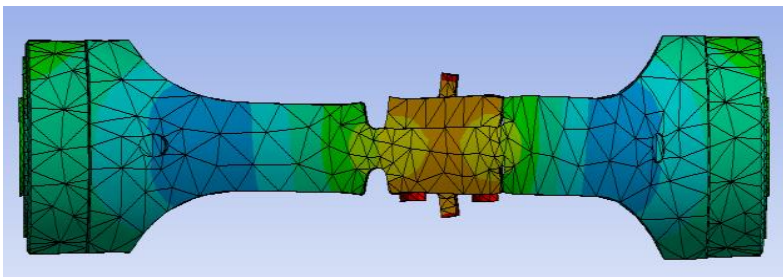

(a) 14th mode, natural frequency $38,326 \mathrm{~Hz}$

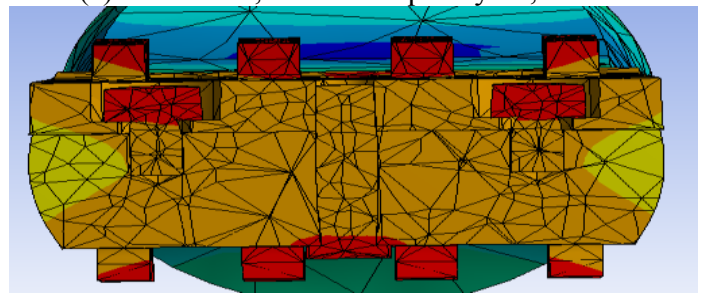

(b) Amplitude distribution

Figure 7. Final FE model and the $14^{\text {th }}$ mode shape

\section{Conclusions}

The welding tips knurled on a horn body directly contact with welding materials and transmit ultrasonic mechanical vibrations, which necessarily cause wears on the tips during ultrasonic welding. If regrinding of worn tips is repeated several times, the mass of the horn is reduced and the frequency characteristic is changed. So, there is a limit to refurbishment. In this study, the tip-replaceable horn of one-wave length in ultrasound is designed by using FE analysis. This horn can allow the part of the tips to be 
attached and detached to/from the horn body. Several models of tip-replaceable horn are proposed. The natural frequencies and vibration modes are investigated through FEA.

As the final model, a horn that only the upper tips are replaceable is proposed, and in the lower part of it, slots are machined to keep the horn as symmetric as possible. This model is modified several times to overcome some problems due to the asymmetry shape of the upper and lower sides of the horn. It is confirmed finally that vibrations occur almost in the axial direction and the natural frequency is similar to that of the conventional one-body horn. From analysis of distributions of vibration amplitude at the cross section along joining bolts, it is shown that vibrational amplitudes are maximum at the tips and are nearly symmetrical in the upper and lower sides. These results imply that the final design for tip-replaceable horn could be applied to ultrasonic welding effectively.

\section{Acknowledgement}

This work (Grants No. C05118390100470156) was supported by Business for R\&D funded The Korea Ministry of SMEs and Startups in 2017.

\section{References}

1. Jiromaru Tsujino, "Ultrasonic welding using complex vibration and high frequency vibration," 2011 IEEE International Ultrasonics Symposium Proceedings (2011), pp. 1910- 1919.
2. Shin-ichi Matsuoka and Hisashi Imai, "Direct welding of different metals used ultrasonic vibration," Journal of Materials Processing Technology 209, pp. 954-960.

3. H. S. Jeong, "Fundamentals of Ultrasonic Welding," Journal of KWS, Vol. 15, No. 6 (1997), pp. 24-31.

4. E. A. NEPPIRAS, "ULTRASONIC WELDING OF METALS,” ULTRASONICS (1965), pp. 128 135 Y.C. Chen, D. Bakavos, A.Gholinia, and P.B. Prangnell, "HAZ development and accelerated post-weld natural ageing in ultrasonic spot welding aluminum 6111-T4 automotive sheet," Acta Materialia 60 (2012), pp. 2816-2828.

5. M. Shakil, N.H. Tariq, M. Ahmad, M.A. Choudhary, J.I. Akhter, and S.S. Babu, "Effect of ultrasonic welding parameters on microstructure and mechanical properties of dissimilar joints," Materials and Design 55 (2014), pp. 263-273.

6. Sang-gon Kim, "Ultrasonic welding horn has several tips," Patent application number 10-2007-0027299.

7. Nissan Motor Co., LTD, "Ultrasonic welding device," International application number PCT/JP2015/073321.

8. BRANSON ULTRASONICS CORPORATION, "Ultrasonic welding device," Japanese patent application number 2013-89327. 